\title{
Targeting CD146 in combination with vorinostat for the treatment of ovarian cancer cells
}

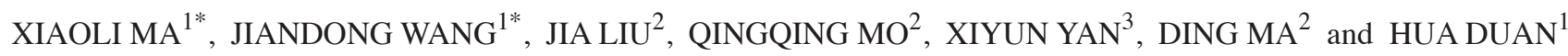 \\ ${ }^{1}$ Gynecological Minimal Invasive Center, Beijing Obstetrics and Gynecology Hospital, \\ Capital Medical University, Beijing 100006; ${ }^{2}$ Cancer Biology Research Center, Tongji Hospital, \\ Tongji Medical College, Huazhong University of Science and Technology, Wuhan, Hubei 430030; \\ ${ }^{3}$ Center of Molecular Biology, Institute of Biophysics, Chinese Academy of Sciences, Beijing 100101, P.R. China
}

Received July 8, 2015; Accepted October 14, 2016

DOI: $10.3892 / 01.2017 .5630$

\begin{abstract}
Drug resistance is the predominant cause of mortality in late-stage patients with ovarian cancer. Histone deacetylase inhibitors (HDACis) have emerged as a novel type of second line drug with high specificity for tumor cells, including ovarian cancer cells. However, HDACis usually exhibit relatively low potencies when used as a single agent. The majority of current clinical trials are combination strategies. These strategies are more empirical than mechanism-based applications. Previously, it was reported that the adhesion molecule cluster of differentiation 146 (CD146) is significantly induced in HDACi-treated tumor cells. The present study additionally confirmed that the induction of CD146 is a common phenomenon in vorinostat-treated ovarian cancer cells. AA98, an anti-CD146 monoclonal antibody (mAb), was used to target CD146 function. Synergistic antitumoral effects between AA98 and vorinostat were examined in vitro and in vivo. The potential effect of combined AA98 and vorinostat treatment on the protein kinase B (Akt) pathway was determined by western blotting. The present study found that targeting of CD146 substantially enhanced vorinostat-induced killing via the suppression of activation of Akt pathways in ovarian cancer cells. AA98 in combination with vorinostat significantly inhibited cell proliferation and increased apoptosis. In vivo, AA98 synergized with vorinostat to inhibit tumor growth and prolong survival in ovarian cancer. These data suggest that an undesired induction of CD146 may serve as a protective response to offset the antitumor efficacy of
\end{abstract}

Correspondence to: Dr Hua Duan, Gynecological Minimal Invasive Center, Beijing Obstetrics and Gynecology Hospital, Capital Medical University, 251 Yao Jiayuan Road, Beijing 100006, P.R. China

E-mail: duanhua888@163.com

${ }^{*}$ Contributed equally

Key words: ovarian cancer, drug resistance, adhesion molecular CD146, vorinostat, Akt, apoptosis vorinostat. By contrast, targeting CD146 in combination with vorinostat may be exploited as a novel strategy to more effectively kill ovarian cancer cells.

\section{Introduction}

Ovarian cancer is one of the most common types of gynecological malignancy and has a poor prognosis. Historically, ovarian cancer was considered a silent cancer, as the majority of patients present with late-stage disease (1). Despite advances in surgery and the development of more effective chemotherapy, ovarian cancer remains the leading cause of mortality from a gynecological cancer (2). Drug resistance is the predominant cause of mortality in late-stage patients. In total, $30 \%$ of patients whose tumors are platinum-resistant will generally either progress during primary therapy or shortly thereafter. Additionally, there is no preferred standard second-line chemotherapy to offer these patients $(3,4)$. Thus, elucidation of mechanisms and identification of new therapeutic targets for ovarian cancer is critical to reduce fatality.

Histone deacetylase inhibitors (HDACis) show promise as a novel class of anticancer agents in a wide spectrum of tumors, including ovarian caner (5). Previously, the present study investigated whether the HDACi trichostatin A (TSA) induces apoptosis of ovarian cancer A2780 cells in a dose-dependent manner (6). Thus far, numerous HDACis are being tested in over 100 clinical trials and have exhibited encouraging therapeutic responses with good safety profiles $(7,8)$. The clinical potential of HDACis has been well documented by the successful development of vorinostat (suberoylanilide hydroxamic acid), which has been approved by the U.S. Food and Drug Administration (9). Despite the rapid progress achieved, clinical data has shown that there is limited efficacy for HDACi as a single agent. The majority of current clinical trials are combination studies looking at HDACi in combination with other agents $(10,11)$. These combination trials seek to increase the antitumor activity of the treatments. Although these combination strategies follow a rational molecular approach in certain cases, in the majority of instances, they are relatively empirical. Accordingly, synergism in antitumor efficacy may be accompanied by adverse effects that are rarely observed with HDACis alone, such 
as severe myelosuppression $(5,12)$. Therefore, revealing the molecular mechanisms underlying the low potency of HDACi is pivotal in determining the optimal application of this class of therapeutic agents in the treatment of ovarian cancer.

In our previous study, it was reported that the adhesion molecule cluster of differentiation 146 (CD146) is significantly induced in HDACi-treated tumor cells (13). In the current study, it was found that the induction of CD146 expression was significant in ovarian cancer cells. CD146 is one of the adhesion molecules belonging to the immunoglobulin superfamily (14). In numerous types of cancer, including melanoma (15), prostate cancer (16) and ovarian cancer, elevated expression of CD146 promotes tumor progression and is associated with poor prognosis. Previously, targeting CD146 with antibody against the molecule has been shown to inhibit tumor growth and angiogenesis in several types of cancer. Based on these findings $(15,17,18)$, the present study chose to additionally explore whether the induced expression of CD146 protected ovarian cancer cells from HDACi-induced death. In addition, the current study tested whether the antitumoral activity of HDACi may be significantly enhanced in combination with the targeting of CD146 in ovarian cancer cells in vitro and in vivo.

\section{Materials and methods}

Cells and reagents. The human ovarian cancer cell lines A2780, SKOV3 and Caov3 were purchased from the American Type Culture Collection (Manassas, VA, USA) and cultured in Dulbecco's modified Eagle's medium containing $10 \%$ fetal calf serum (Gibco; Thermo Fisher Scientific, Inc., Waltham, MA, USA). All cells were cultured at $37^{\circ} \mathrm{C}$ in a humidified $5 \% \mathrm{CO}_{2}$ atmosphere. The HDACi TSA and vorinostat were purchased from Sigma-Aldrich (Merck Millipore, Darmstadt, Germany), and dissolved in dimethyl sulfoxide (DMSO). The mouse anti-human CD146 monoclonal antibody (mAb) AA98 and the control mIgG were provided by Dr Xiyun Yan (Institute of Biophysics, Chinese Academy of Sciences, Beijing, China) (19). The mouse anti-human CD146 mAb (1 mg/ml; ab24577) was purchased from Abcam (Cambridge, UK). Fluorescein isothiocyanate-conjugated mouse anti-human CD146 mAb (1:100; 11-1469-42) was purchased from eBioscience, Inc. San Diego, CA, USA). Anti protein kinase B (Akt) rabbit anti-human polyclonal antibody (1:1,000; \#9272S), anti-phosphorylated Akt rabbit anti-human $\mathrm{mAb}(1: 1,000$; \#4058), anti-human phosphorylated glycogen synthase kinase $3 \beta$ (GSK3 $\beta$ ) rabbit mAb, (1:1,000; \#5558), anti-human phosphorylated 4E-binding protein 1 (4E-BP1) rabbit $\mathrm{mAb}(1: 1,000$; \#2855) and anti-human phosphorylated ribosomal protein S6 kinase-1 (S6K1) mouse mAbs (1:1,000; \#9206) were purchased from Cell Signaling Technology, Inc. (Danvers, MA, USA). Triciribine was purchased from Cayman Chemical Company (Ann Arbor, MI, USA). Full-length Akt2 complementary DNA (cDNA) was cloned into pcDNA3.1 plasmid and termed the AAkt2 vector, which has been described previously (13).

Cell viability assays. Cell viability was determined using a MTT assay. In brief, $5 \times 10^{3}$ cells were plated into each well of 96-well plates for $72 \mathrm{~h}$ following the indicated treatments.
Subsequently, $5 \mathrm{mg} / \mathrm{ml} \mathrm{MTT}$ was added and incubated at $37^{\circ} \mathrm{C}$ for $4 \mathrm{~h}$. The medium was then removed, and $1 \mathrm{ml}$ DMSO was added to solubilize the MTT-formazan product. The MTT absorbance was then determined at $570 \mathrm{~nm}$ on a Multiscan JX ver1.1 (Thermo Labsystems, Santa Rosa, CA, USA). Results are expressed as a percentage of the viable cells in the DMSO-treated group. Each data point is the mean \pm standard error of the mean of 6 replicates.

Apoptosis assays. Cells were stained with Annexin V and propidium iodide and the percentage of apoptotic cells were determined by flow cytometry, as described previously (20). CELL Quest software (BD Biosciences, Franklin Lakes, NJ, USA) was used for data acquisition and analysis.

Quantitative polymerase chain reaction $(q P C R)$. Total RNA was isolated from A2780 or SKOV3 cells after vorinostat treatment using TRIzol reagent (Invitrogen; Thermo Fisher Scientific, Inc.), according to the manufacturer's instructions. RNA quantitation was determined using a NanoDrop micro-volume spectrophotometer (Thermo Fisher Scientific, Inc.), and the messenger RNA (mRNA) integrity was verified by agarose gel electrophoresis. Reverse transcription (RT)-qPCR was then performed on $2 \mu \mathrm{g}$ total RNA using a PrimeScript RT Reagent kit with gDNA Eraser (Takara Bio, Inc., Otsu, Japan). qPCR was performed in ABI Prism 7000 (Applied Biosystems; Thermo Fisher Scientific, Inc.) with the SYBR Green PCR Master Mix (Sigma-Aldrich; Merck Millipore) using the following thermocycler program for all genes: $5 \mathrm{~min}$ of pre-incubation at $95^{\circ} \mathrm{C}$, followed by 40 cycles of $15 \mathrm{sec}$ at $95^{\circ} \mathrm{C}, 15 \mathrm{sec}$ at $60^{\circ} \mathrm{C}$, and $30 \mathrm{sec}$ at $72^{\circ} \mathrm{C}$. The primers for were as follows: CD146 forward, 5'-CAG TCCTCATACCAGAGCCAACAG-3' and reverse, 5'-GGA CCAGGATGCACACAATCA-3'; and 18S ribosomal RNA forward, 5'-AGTCCCTGCCCTTTGACACA-3' and reverse, 5'-GATCCGAGGGCCTCACTAAAC-3'. The 18S ribosomal RNA was used as an internal control. All primers were obtained from Tiangen Biotech Co., Ltd. (Beijing, China). A melting curve assay was performed to determine the purity of the amplified product. Contamination with genomic DNA was not detected in any of the analyzed samples. Each sample was assayed in triplicate, analysis of relative gene expression data used the $2^{-\Delta \Delta C q}$ method, as previously described (21), and the results were expressed as fold induction compared with the untreated group.

Western blot analysis. Detection of the CD146, AKT, p-AKT, P-4E-BP1, p-S6K1, p-GSK-3 $\beta$ and $\beta$-actin by SDS-PAGE was performed as previously described (21).

Soft agar colony-forming assay. Cells were treated with $10 \mu \mathrm{g} / \mathrm{ml} \mathrm{AA98,} 2.5 \mu \mathrm{mol} / 1$ vorinostat or vorinostat + AA98 for $24 \mathrm{~h}$. DMSO-treated cells were used as a negative control. A total of $1 \times 10^{3}$ cells were then plated in $60-\mathrm{mm}$ culture plates in medium containing $0.3 \%$ agar overlying a $0.5 \%$ agar layer. The cells were subsequently incubated for 14 days at $37^{\circ} \mathrm{C}$ and colonies were stained with $0.5 \mathrm{ml}$ of $0.0005 \%$ crystal violet solution for $1 \mathrm{~h}$ and counted using a dissecting microscope (x50 magnification). The results are expressed as a percentage of colonies in the DMSO-treated group. 
Animal experiments. In total, 120 female athymic BALB/c nude mice were obtained from the Animal Center of the Chinese Academy of Medical Science (Beijing, China). The 6-week-old mice used were maintained in a laminar-flow cabinet under specific pathogen free conditions. In tumor xenograft models, $1 \times 10^{7}$ SKOV3 cells were injected subcutaneously. Once tumors had grown between 5 and $6 \mathrm{~mm}$, the mice were grouped $(n=10)$ and administered intraperitoneally with $8 \mathrm{mg} / \mathrm{kg}$ of AA98 or $20 \mathrm{mg} / \mathrm{kg}$ of vorinostat or vorinostat + AA98 twice a week until the mice were sacrificed (tumor volume $>1,000 \mathrm{~mm}^{3}$ or 42 days subsequent to treatment). PBS served as a control. Tumor size was determined twice a week and tumor volume was determined according to the equation: Tumor size $\left(\mathrm{cm}^{3}\right)=$ width $^{2}$ xlengthx $(\pi / 6)$.

Laser scanning cytometry (LSC). LSC slides were scanned using an LSC instrument equipped with argon (Ar; $488 \mathrm{~nm}$ ) and helium-neon (HeNe; $633 \mathrm{~nm}$ ) laser and iCys3.3.4 software (CompuCyte; Beckman Coulter, Inc., Brea, CA, USA). DNA staining based on hematoxylin served as the trigger/contouring parameter. The following channels and settings were used for data collection: Argon green photomultiplier tube (PMT, 15-25\%; offset, 0.2; gain, 13\%) and HeNe LongRed (LR; PMT, 14-22\%; offset, 0-0.3; gain, 13\%). The present study analyzed the immunohistochemical tissue samples in phantom mode. Argon green and HeNe LongRed parameters were collected with aberration compensation. Statistical analysis was performed on the results of 3 independent experiments using the paired Student's t-test.

Statistical analysis. The statistical significance of differences between experimental and control groups was determined by one-way analysis of variance followed by the Student-Newman-Keuls test using SPSS software version 13.0 (SPSS, Inc., Chicago, IL, USA). All statistical tests were two sided, and $\mathrm{P}<0.05$ was considered to indicate a statistically significant difference. Statistical analysis of LSC findings was performed on the results of 3 independent experiments using a paired Student's $t$ test.

\section{Results}

Induction of adhesion molecular CD146 is a common phenomenon in vorinostat-treated ovarian cancer cells in vitro and in vivo. In previous studies, adhesion molecule CD146 was observed to be significantly upregulated following $\mathrm{HDACi}$ treatment in ovarian cancer cells (13). In addition, previous studies have linked CD146 with apoptosis resistance in cancer cells $(21,22)$. To additionally verify whether expression of CD146 is induced by vorinostat, the present study investigated the effects of vorinostat on mRNA and protein expression of CD146 in ovarian cancer cells. A2780 and SKOV3 cells were treated with $2.5 \mu \mathrm{mol} / 1$ vorinostat for $12 \mathrm{~h}$. As shown in Fig. 1A, subsequent to treatment with vorinostat, transcriptional induction of CD146 reached an extremely high level, 468.5 fold for A2780 and 450.3 fold for SKOV3 $(\mathrm{P}<0.001)$, compared with the basal transcriptional level.

Furthermore, another HDACi, TSA, significantly induced the expression of CD146, indicating that the induction of CD146 expression may be a common action shared by HDACi.
To determine whether the vorinostat-induced expression of CD146 occurs in primary ovarian cancer cells, 8 primary tumor samples from patients with ovarian cancer were treated with vorinostat. Vorinostat significantly induced the expression of CD146 as early as $3 \mathrm{~h}$ subsequent to treatment and the increase lasted up to $12 \mathrm{~h}$ in all of the samples examined (Table I). To test whether the induction of CD146 transcription upregulated the level of CD146 protein, cultured A2780 cells were treated with vorinostat or DMSO and examined for CD146 protein expression using immunofluorescence and western blotting. As expected, treatment with vorinostat significantly enhanced the positive immunoreactivity and protein level of CD146 in A2780 cells (Fig. 1B and C).

To address whether the induction of CD146 occurs in vivo, SKOV3 tumor-bearing mice $(n=10)$ were treated with vorinostat at $20 \mathrm{mg} / \mathrm{kg}$ based on earlier studies (23). Similarly, CD146 expression was markedly elevated in the tumor cell membrane $24 \mathrm{~h}$ subsequent to treatment with vorinostat, as determined by immunohistochemistry and quantified by LSC (Fig. 1D); the total positive rate for CD146 in SKOV3 tumors treated with vorinostat compared with those treated with DMSO was $40 \pm 2$ vs. $30 \pm 1 \%$, $(\mathrm{P}=0.001)$.

Targeting CD146 substantially enhanced vorinostat-induced killing in ovarian cancer cells. To additionally confirm whether knockdown of CD146 enhanced vorinostat-induced cell death in ovarian cancer cells, A2780 ovarian cancer cells were cultured with DMSO or AA98, which has been confirmed to significantly knockdown the expression of CD146 (Fig. 2A). A2780/SKOV3/Caov3 ovarian cancer cells are exposed to $2.5 \mu \mathrm{mol} / 1$ vorinostat for $72 \mathrm{~h}$ and subjected to apoptosis assay for the determination of their drug sensitivity. Accordingly, knockdown of CD146 increased the sensitivity of A2780/SKOV3/Caov3 ovarian cancer cells to vorinostat-induced apoptosis (Fig. 2B; A2780, 18.7 \pm 3.6 vs. $49.06 \pm 4.3 \%, \mathrm{P}=0.001$; SKOV3, $16.28 \pm 2.9$ vs. $38.13 \pm 3.5 \%$, $\mathrm{P}=0.001)$. Furthermore, knockdown of CD146 promoted vorinostat-induced killing and gave rise to less survival colonies in A2780 cells and SKOV3 cells (Fig. 2C and D; A2780, $46.73 \pm 5.2$ vs. $19.16 \pm 6.3 \%, \mathrm{P}=0.004$; $\mathrm{SKOV} 3,37.55 \pm 3.6$ vs. $16.23 \pm 2.4 \%, \mathrm{P}=0.001)$.

Knockdown of CD146 promotes vorinostat-induced apoptosis via suppression of the Akt pathway in ovarian cancer cells. Data has previously shown a link between CD146 expression and Akt activation (24); therefore, the present study sought to determine the effects of vorinostat/AA98 on the Akt pathway in ovarian caner cells A2780. Vorinostat induced the phosphorylation of Akt and its downstream targets 4E-BP1 and S6K1. AA98 co-administration with vorinostat reverses the activation of the Akt pathway induced by vorinostat (Fig. 3A). To additionally confirm whether Akt had a protective effect on vorinostat/AA98-induced apoptosis, overexpression/inhibition experiments were performed using AAkt2 plasmid transfection or triciribine treatment. Although transfection of AAkt2 inhibited vorinostat/AA98-induced apoptosis, the inhibition of Akt phosphorylation by triciribine substantially sensitized A2780 cells to vorinostat/AA98-induced killing (Fig. 3B, control vs. AAKT2, P=0.01; Fig. 3C, control vs. triciribine, $\mathrm{P}=0.01$ ). 
Table I. Effect of vorinostat on the expression of CD146 in clinical tumor samples.

Time course, $\mathrm{h}$

\begin{tabular}{lllllll}
\cline { 4 - 6 } Patients & Clinical diagnosis & Classification & 0 & 3 & 6 & 12 \\
\hline Patient 1 & Ovarian cancer & Serous & 1 & $76.40 \pm 7.25$ & $16.33 \pm 2.78$ & $15.97 \pm 1.64$ \\
Patient 2 & Ovarian cancer & Mucinous & 1 & $77.13 \pm 6.65$ & $14.02 \pm 2.45$ & $13.23 \pm 1.76$ \\
Patient 3 & Ovarian cancer & Serous & 1 & $80.52 \pm 7.81$ & $18.59 \pm 2.97$ & $17.13 \pm 2.35$ \\
Patient 4 & Ovarian cancer & Serous & 1 & $54.21 \pm 4.36$ & $15.47 \pm 3.75$ & $11.24 \pm 1.88$ \\
Patient 5 & Ovarian cancer & Mucinous & 1 & $73.26 \pm 6.82$ & $21.67 \pm 1.98$ & $20.57 \pm 1.18$ \\
Patient 6 & Ovarian cancer & Serous & 1 & $103.42 \pm 8.93$ & $46.15 \pm 2.60$ & $37.22 \pm 2.71$ \\
Patient 7 & Ovarian cancer & Serous & 1 & $66.57 \pm 5.33$ & $50.14 \pm 3.08$ & $26.89 \pm 3.43$ \\
Patient 8 & Ovarian cancer & Serous & 1 & $90.36 \pm 7.47$ & $65.11 \pm 4.23$ & $30.78 \pm 3.59$
\end{tabular}

Data are expressed as fold increase (mean \pm standard error) of CD146 messenger RNA from vorinostat-treated ovarian cancer cells relative to that from medium-treated cells. Each data point was obtained from $\geq 3$ independent experiments. CD146, cluster of differentiation 146 .
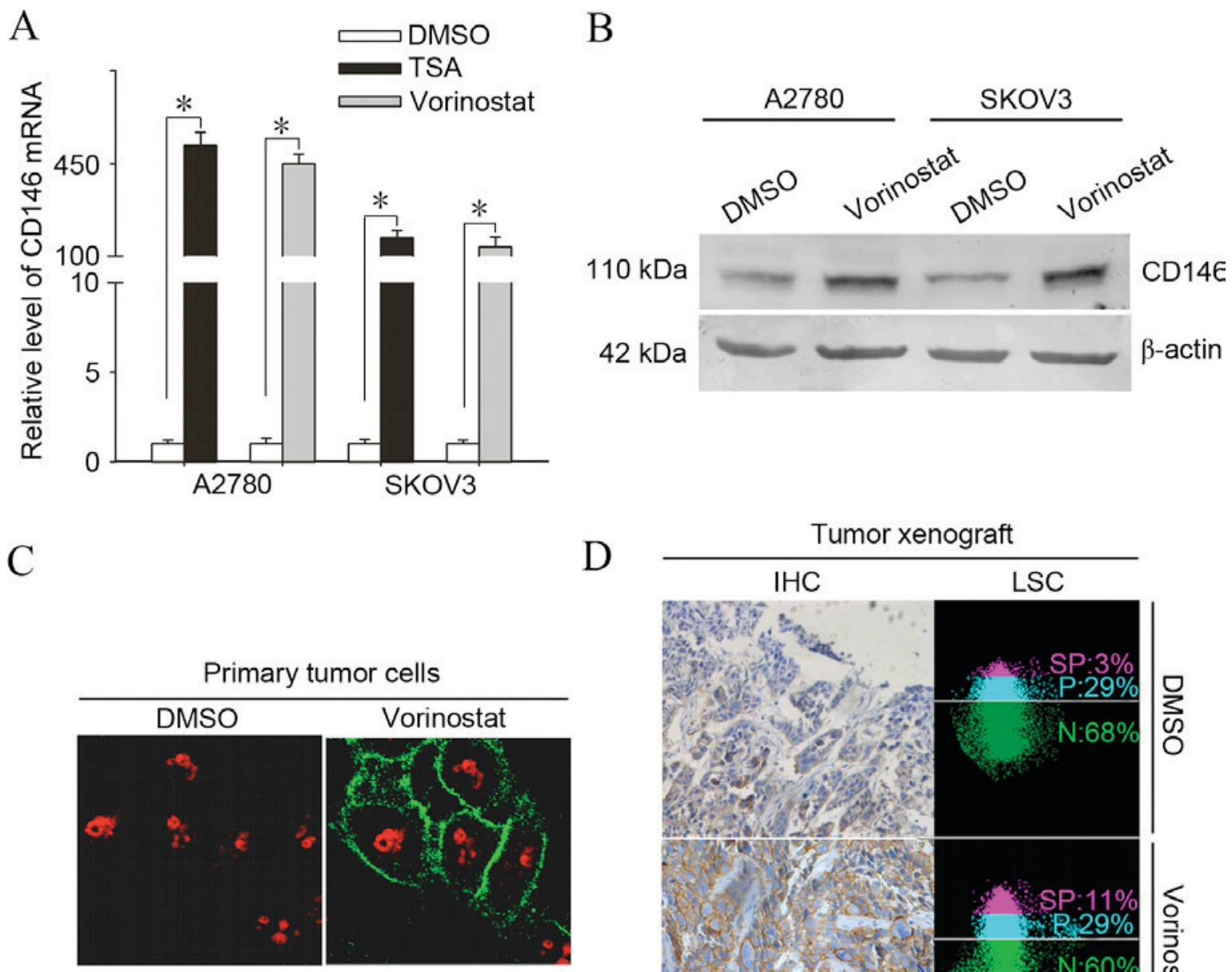

$\mathrm{D}$

Tumor xenograft

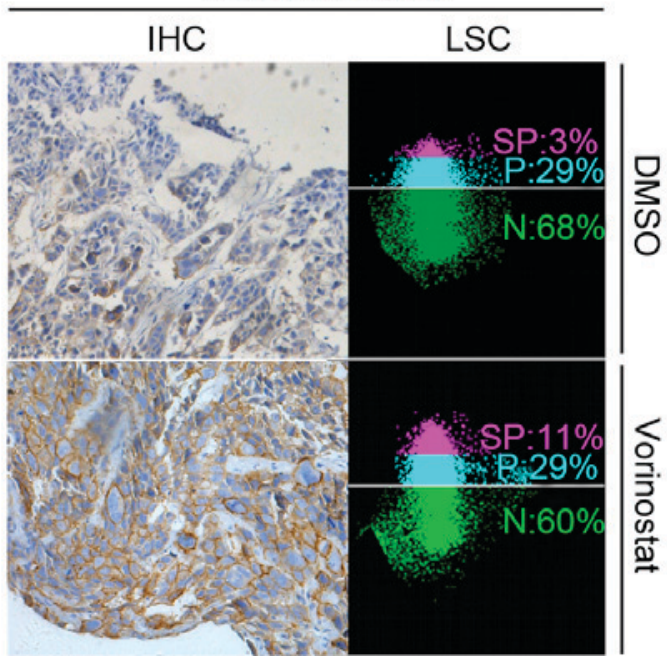

Figure 1. Induction of the adhesion molecule CD146 is a common phenomenon in vorinostat-treated ovarian cancer cells in vitro and in vivo. (A) A2780 and SKOV3 cells were treated with $2.5 \mu \mathrm{mol} / 1$ vorinostat or $500 \mathrm{nmol} / 1 \mathrm{TSA}$ for $12 \mathrm{~h}$ and subjected to analysis of quantitative polymerase chain reaction for the mRNA levels of CD146. Results are normalized to those of $18 \mathrm{~s}$ RNA and expressed as the fold induction compared with the DMSO-treated group ( $\mathrm{P}<0.05$ ). (B) A2780 and SKOV3 cells were treated with $2.5 \mu \mathrm{mol} / 1$ vorinostat for $24 \mathrm{~h}$ and analyzed for the protein levels of CD146 by western blot analysis. (C) A2780 cells were treated with $2.5 \mu \mathrm{mol} / 1$ vorinostat or DMSO for $12 \mathrm{~h}$ and were then analyzed by immunofluorescent analysis for staining of CD146, obtaining representative images under a confocal microscope (magnification, x600). (D) SKOV3 tumor-bearing mice were treated with 25 mg/kg of vorinostat or DMSO for $24 \mathrm{~h}$ and CD146 expression was determined by immunohistochemistry and quantified by laser scanning cytometry. Images represent typical data (Total positive rate for CD146 (SP plus P) is the mean \pm standard deviation ( $\mathrm{n}=10$ ). CD146, cluster of differentiation 146; DMSO, dimethyl sulfoxide; TSA, trichostatin A; SP, strong positive; P, positive; N, negative; IHC, immunohistochemistry; mRNA, messenger RNA; LSC, laser scanning cytometry. 
A

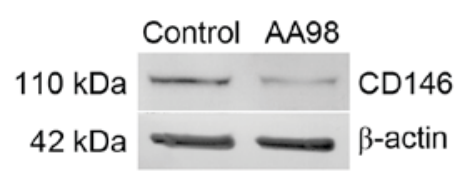

$\mathrm{C}$

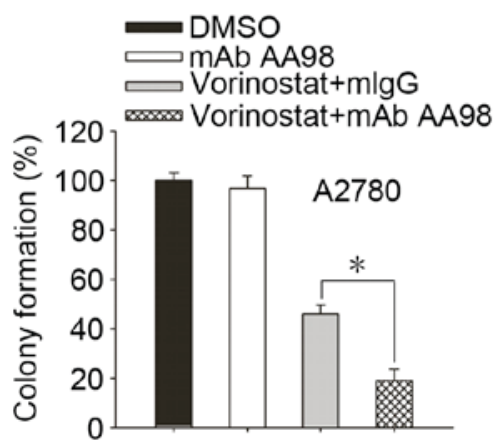

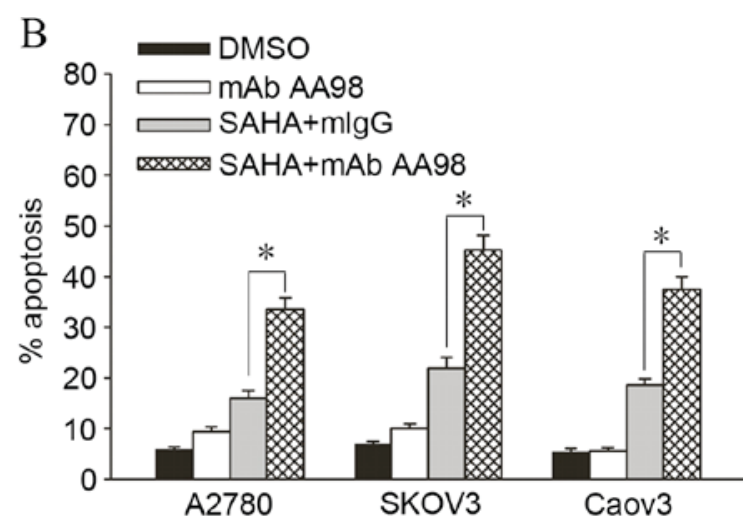

$\mathrm{D}$

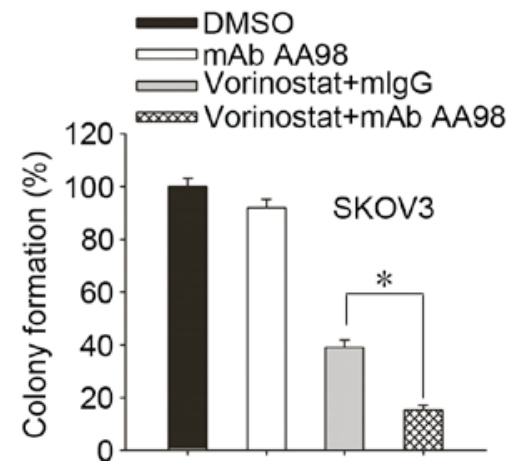

Figure 2. Targeting CD146 substantially enhanced vorinostat-induced killing in ovarian cancer cells. (A) Ovarian cancer A2780 cells were cultured with control $\mathrm{mAb} \mathrm{mIgG}$ or AA98, which has been confirmed to significantly knockdown the expression of CD146 by western blot analysis. (B) A2780/SKOV3/Caov3 ovarian cancer cells were exposed to $2.5 \mu \mathrm{mol} / 1$ vorinostat for $72 \mathrm{~h}$ and subjected to apoptosis assay for the determination of their drug sensitivity, where each data point represents the mean \pm standard error of the mean of 3 replicates. (C) A2780 or (D) SKOV3 cells were treated with $10 \mu \mathrm{g} / \mathrm{ml} \mathrm{mAb}$ AA98 or 2.5 $\mu \mathrm{mol} / 1$ vorinostat + isotype-matched $\mathrm{mIgG}$, or vorinostat $+\mathrm{mAb}$ AA98 for $24 \mathrm{~h}$ and then subjected to the soft agar colony-forming assay ("P<0.05). Results are expressed as a percentage of colonies in the DMSO-treated group. Each data point represents the mean \pm standard deviation of 3 replicates. CD146, cluster of differentiation 146; mAb, monoclonal antibody; $\mathrm{mIgG}$, monoclonal immunoglobulin G; DMSO, dimethyl sulfoxide; SAHA, suberoylanilide hydroxamic acid.

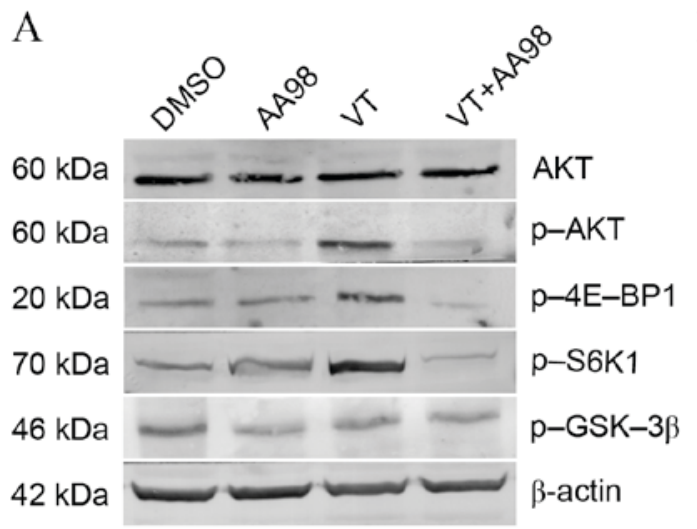

B

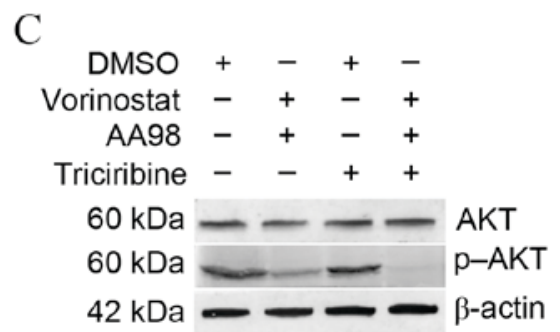

Figure 3. Knockdown of CD146 promotes vorinostat-induced apoptosis via suppression of the Akt pathway in ovarian cancer cells. (A) A2780 cells were treated as depicted for $24 \mathrm{~h}(5 \mu \mathrm{mol} / 1$ vorinostat; $10 \mu \mathrm{g} / \mathrm{ml} \mathrm{mAb} \mathrm{AA98)}$ and examined for protein levels of total Akt, p-Akt, P-4E-BP1, p-S6K1, p-GSK-3 $\beta$ and $\beta$-actin. VT analysis by western blotting. (B) A2780 cells stably transfected with the AAkt 2 plasmid were treated as depicted for $24 \mathrm{~h}$ and examined for protein levels of total Akt and p-Akt by western blot analysis. A2780 cells stably transfected with pcDNA3.1 plasmid were treated as control group. (C) A2780 cells were treated with as depicted for $24 \mathrm{~h}(5 \mu \mathrm{mol} / 1$ vorinostat; $10 \mu \mathrm{g} / \mathrm{ml} \mathrm{mAb} \mathrm{AA98;} 5 \mu \mathrm{mol} / 1$ triciribine $)$ and examined for protein levels of total Akt and p-Akt by western blot analysis. CD146, cluster of differentiation 146; Akt, protein kinase B; p-, phosphorylated; 4E-BP1, 4E-binding protein 1; S6K1, ribosomal protein S6 kinase-1; GSK-3 $\beta$, glycogen synthase kinase 3 $\beta$; VT, vorinostat treatment; DMSO, dimethyl sulfoxide. 
A

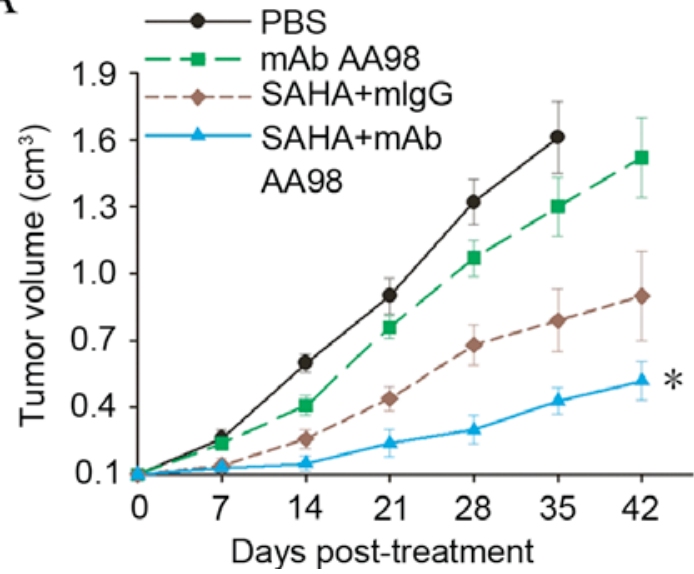

$\mathrm{B}$

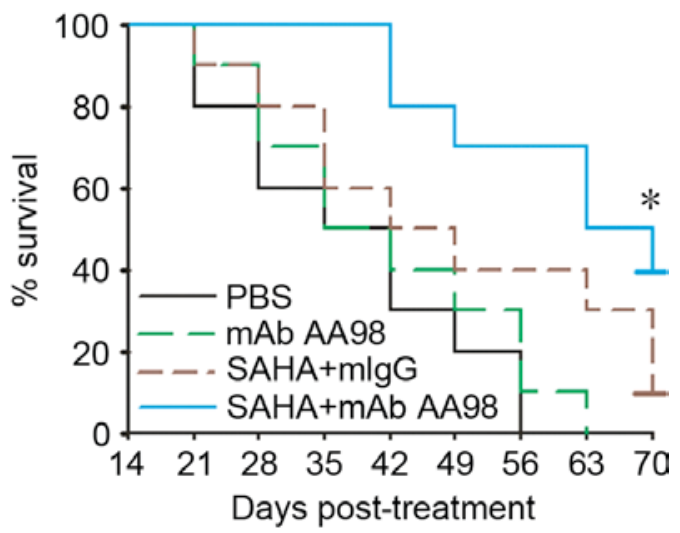

Figure 4. Targeting CD146 synergized with vorinostat to substantially inhibit ovarian cancer growth. (A) When the tumor reached a diameter of 5-6 mm, SKOV3 tumor-bearing mice were grouped ( $\mathrm{n}=10)$ and administered intraperitoneally with PBS (black), 8 mg/kg mAb AA98 (green), 20 mg/kg vorinostat plus mIgG (brown), or vorinostat plus mAb AA98 (blue). Mean tumor volumes were monitored at specific time points subsequent to treatment. Each data point represents mean \pm standard deviation $\left({ }^{*} \mathrm{P}<0.05 ; \mathrm{n}=10\right)$. (B) Kaplan-Meier survival curves of SKOV3 tumor-bearing mice following various treatments as indicated. Each group consisted of 10 animals. PBS served as a control. CD146, cluster of differentiation 146; mAb, monoclonal antibody; mIgG, monoclonal immunoglobulin G; SAHA, suberoylanilide hydroxamic acid.

Targeting CD146 synergized with vorinostat to substantially inhibit ovarian cancer growth. To determine the in vivo antitumor efficacy of combined vorinostat and AA98, the present study chose lower doses of the two agents compared with those previously reported (13). The animal study was completed when the tumor reached a diameter of 5-6 $\mathrm{mm}$. The SKOV3 tumor-bearing mice were grouped $(n=10)$ and administered intraperitoneally with AA98 or vorinostat. Although no tumor complete regression was observed in any groups with different treatments, tumor growth was significantly retarded in the group with combined vorinostat and AA98 treatment $(\mathrm{P}=0.02$; Fig. 4A). Furthermore, combined vorinostat and AA98 treatment significantly improved the survival rate in SKOV3 tumor-bearing mice $(\mathrm{P}=0.001$; Fig. 4B).

\section{Discussion}

The majority of patients with ovarian cancer have progressed to advanced stages of disease by the first clinical visit, and are therefore not eligible to be treated with surgery, and can only receive chemotherapy, with poor results (25). Drug resistance is the primary cause of mortality in late-stage patients. The flood of new second line drugs in previous years has provided numerous marked improvements in anticancer therapy (26). Thus, the development of new therapeutic strategies and search for novel genes with new mechanisms of action that can lead to drug resistance of ovarian cancer cell have become the focuses of current cancer research.

HDACis have emerged as novel second line drugs, with their high specificity for tumor cells. However, since the targets of HDACis are so extensive, it is not surprising that HDACis would initiate anti-apoptotic and pro-apoptotic therapeutic responses. HDACis usually exhibit relatively low potency when used as single agents. The majority of the current HDACi combination strategies are more empirical than mechanism-based applications, and accordingly, are not optimal for this class of drugs $(27,28)$. In our previous study, a cDNA microarray analysis was conducted and it was found that the expression of adhesion molecule CD146 was significantly induced in HDACi-treated tumor cells, particularly in ovarian cancer cells (13). In the present findings, it was verified that the induction of CD146 is a common phenomenon in vorinostat-treated ovarian cancer cells in vitro and in vivo. Targeting CD146 substantially sensitized ovarian cancer cells to vorinostat-induced killing. Treatment with vorinostat plus AA98 also preferentially inhibits cell proliferation, enhances apoptotic rate of ovarian cancer cells and ablates cancer colony formation. The present findings provide the first evidence that an undesired protective signal is initiated by HDACi and highlight a novel molecular mechanism by which HDACi induces the expression of CD146 as a protective response to offset the antitumor efficacy. By contrast, the induction of CD146 may be exploited as a novel strategy for the enhanced killing of ovarian cancer cells. Similarly, the synergistic killing effect of vorinostat and targeting of CD146 was observed in vivo. Treatment of SKOV3 xenografts with vorinostat plus AA98 resulted in a more pronounced decrease in tumor volume compared with single drug-treated mice. Additionally, to inhibit tumor growth, it was shown that the combined regimen of vorinostat and AA98 is able to significantly prolong the survival rate of tumor-bearing mice.

It is well known that the sensitivity of cancer cells to chemotherapeutic drug-induced apoptosis depends on the balance between pro-apoptotic and anti-apoptotic signals (29). Therefore, inhibition of anti-apoptotic signals, such as those mediated by the Akt pathway, has been proposed as a promising strategy to enhance the efficacy of chemotherapeutic agents (30). The present data show that the increased sensitivity to vorinostat caused by AA98 was strongly associated with Akt signaling in ovarian carcinomas. The combination of vorinostat with AA98 attenuates Akt phosphorylation and 4E-BP1 expression. A similar association has been reported between Akt activation and HDACi sensitivity in cervical cancer cell lines (31). However, Akt kinase activity is not the sole determinant of sensitivity to vorinostat, and certain factors, such as S6K1, can result in sensitivity to vorinostat in 
the absence of Akt activation (32). In ovarian cancer A2780 cells, AA98 co-administration reverses the activation of S6K1 induced by vorinostat. Furthermore, it was confirmed that Akt had a protective effect on vorinostat/AA98-induced apoptosis by overexpression/inhibition experiments.

Collectively, targeting CD146 may be exploited as a novel strategy to more effectively kill ovarian cancer cells. However, the identification of an optimal HDACi-based regimen requires long-term and painstaking clinical trials and suboptimal application. The current preclinical approach may accelerate the design of an optimal HDACi-containing regimen in the treatment of ovarian cancer.

\section{Acknowledgements}

The present study was supported by a grant from the Beijing Nova Program (grant no. Z141107001814015), National Natural Science Foundation of China (grant no. 81101970) and $\mathrm{PhD}$ Programs Foundation of Ministry of Education of China (grant no. 20111107120009).

\section{References}

1. Suh DH, Lee KH, Kim K, Kang S and Kim JW: Major clinical research advances in gynecologic cancer in 2014. J Gynecol Oncol 26: 156-167, 2015.

2. Jayson GC, Kohn EC, Kitchener HC and Ledermann JA: Ovarian cancer. Lancet 384: 1376-1388, 2014.

3. Zahedi P, Yoganathan R, Piquette-Miller M and Allen C: Recent advances in drug delivery strategies for treatment of ovarian cancer. Expert Opin Drug Deliv 9: 567-583, 2012.

4. Vecchione A, Belletti B, Lovat F, Volinia S, Chiappetta G, Giglio S, Sonego M, Cirombella R, Onesti EC, Pellegrini P, et al: A microRNA signature defines chemoresistance in ovarian cancer through modulation of angiogenesis. Proc Natl Acad Sci USA 110: 9845-9850, 2013

5. Zwergel C, Valente S, Jacob C and Mai A: Emerging approaches for histone deacetylase inhibitor drug discovery. Expert Opin Drug Discov 10: 599-613, 2015.

6. Ma XL, Duan H, Liu J, Mo Q, Sun C, Ma D and Wang J: Effect of LIV1 on the sensitivity of ovarian cancer cells to trichostatin A. Oncol Rep 33: 893-898, 2015.

7. West AC and Johnstone RW: New and emerging HDAC inhibitors for cancer treatment. J Clin Invest 124: 30-39, 2014.

8. Slingerland M, Guchelaar HJ and Gelderblom H: Histone deacetylase inhibitors: An overview of the clinical studies in solid tumors. Anticancer Drugs 25: 140-149, 2014.

9. Højfeldt JW, Agger K and Helin K: Histone lysine demethylases as targets for anticancer therapy. Nat Rev Drug Discov 12 917-930, 2013

10. Huang Z, Peng S, Knoff J, Lee SY, Yang B, Wu TC and Hung CF: Combination of proteasome and HDAC inhibitor enhances HPV16 E7-specific CD8+ T cell immune response and antitumor effects in a preclinical cervical cancer model. J Biomed Sci 22: 7, 2015.

11. Straus DJ, Hamlin PA, Matasar MJ, Lia Palomba M, Drullinsky PR, Zelenetz AD, Gerecitano JF, Noy A Hamilton AM, Elstrom R, et al: Phase I/II trial of vorinostat with rituximab, cyclophosphamide, etoposide and prednisone as palliative treatment for elderly patients with relapsed or refractory diffuse large B-cell lymphoma not eligible for autologous stem cell transplantation. Br J Haematol 168: 663-670, 2015.

12. Rasheed WK, Johnstone RW and Prince HM: Histone deacetylase inhibitors in cancer therapy. Expert Opin Investig Drugs 16 659-678, 2007

13. Ma X, Liu J, Wu J, Yan X, Wu P, Liu Y, Li S, Tian Y, Cao Y, Chen $\mathrm{G}$, et al: Synergistic killing effect between vorinostat and target of CD146 in malignant cells. Clin Cancer Res 16: $5165-5176,2010$
14. Wang Z and Yan X: CD146, a multi-functional molecule beyond adhesion. Cancer Lett 330: 150-162, 2013.

15. Xie S, Luca M, Huang S, Gutman M, Reich R, Johnson JP and Bar-Eli M: Expression of MCAM/MUC18 by human melanoma cells leads to increased tumor growth and metastasis. Cancer Res 57: 2295-2303, 1997.

16. Wu GJ, Wu MW, Wang SW, Liu Z, Qu P, Peng Q, Yang H, Varma VA, Sun QC, Petros JA, et al: Isolation and characterization of the major form of human MUC18 cDNA gene and correlation of MUC18 over-expression in prostate cancer cell lines and tissues with malignant progression. Gene 279: 17-31, 2001.

17. Zabouo G, Imbert AM, Jacquemier J, Finetti P, Moreau T, Esterni B, Birnbaum D, Bertucci F and Chabannon C: CD146 expression is associated with a poor prognosis in human breast tumors and with enhanced motility in breast cancer cell lines. Breast Cancer Res 11: R1, 2009.

18. Leslie MC, Zhao YJ, Lachman LB, Hwu P, Wu GJ and Bar-Eli M: Immunization against MUC18/MCAM, a novel antigen that drives melanoma invasion and metastasis. Gene Ther 14: 316-323, 2007.

19. Xing S, Luo Y, Liu Z, Bu P, Duan H, Liu D, Wang P, Yang J, Song L, Feng J, et al: Targeting endothelial CD146 attenuates colitis and prevents colitis-associated carcinogenesis. Am J Pathol 184: 1604-1616, 2014.

20. Ma XL, Ma QF, Liu J, Tian Y, Wang B, Taylor KM, Wu P, Wang D, Xu G, Meng L, et al: Identification of LIV1, a putative zinc transporter gene responsible for HDACi-induced apoptosis, using a functional gene screen approach. Mol Cancer Ther 8: 3108-3116, 2009.

21. Satyamoorthy K, Muyrers J, Meier F, Patel D and Herlyn M: Mel-CAM-specific genetic suppressor elements inhibit melanoma growth and invasion through loss of gap junctional communication. Oncogene 20: 4676-4684, 2001.

22. Lei X, Guan CW, Song Y and Wang H: The multifaceted role of CD146/MCAM in the promotion of melanoma progression. Cancer Cell Int 15: 3, 2015.

23. Cooper AL, Greenberg VL, Lancaster PS, van Nagell JR Jr, Zimmer SG and Modesitt SC: In vitro and in vivo histone deacetylase inhibitor therapy with suberoylanilide hydroxamic acid (SAHA) and paclitaxel in ovarian cancer. Gynecol Oncol 104: 596-601, 2007.

24. Zeng Q, Wu Z, Duan H, Jiang X, Tu T, Lu D, Luo Y, Wang P, Song L, Feng J, et al: Impaired tumor angiogenesis and VEGF-induced pathway in endothelial CD146 knockout mice. Protein Cell 5: 445-456, 2014.

25. Lheureux S, Karakasis K, Kohn EC and Oza AM: Ovarian cancer treatment: The end of empiricism? Cancer 121: 3203-3211, 2015.

26. Modugno F and Edwards RP: Ovarian cancer: Prevention, detection, and treatment of the disease and its recurrence. molecular mechanisms and personalized medicine meeting report. Int J Gynecol Cancer 22: S45-S57, 2012.

27. Bots $\mathrm{M}$ and Johnstone RW: Rational combinations using HDAC inhibitors. Clin Cancer Res 15: 3970-3977, 2009.

28. Khabele D: The therapeutic potential of class I selective histone deacetylase inhibitors in ovarian cancer. Front Oncol 20: 111, 2014.

29. Mohammad RM, Muqbil I, Lowe L, Yedjou C, Hsu HY, Lin LT, Siegelin MD, Fimognari C, Kumar NB, Dou QP, et al: Broad targeting of resistance to apoptosis in cancer. Semin Cancer Biol 35 (Suppl): S78-S103, 2015.

30. Ocana A, Vera-Badillo F, Al-Mubarak M, Templeton AJ, Corrales-Sanchez V, Diez-Gonzalez L, Cuenca-Lopez MD, Seruga B, Pandiella A and Amir E: Activation of the $\mathrm{PI} 3 \mathrm{~K} / \mathrm{mTOR} / \mathrm{AKT}$ pathway and survival in solid tumours: Systematic review and meta-analysis. PLoS One 9, e95219, 2014.

31. Feng D, Cao Z, Li C, Zhang L, Zhou Y, Ma J, Liu R, Zhou H, Zhao W, Wei H and Ling B: Combination of valproic acid and ATRA restores RAR $\beta 2$ expression and induces differentiation in cervical cancerthrough the PI3K/Akt pathway. Curr Mol Med 12: 342-354, 2012.

32. Moschetta M, Reale A, Marasco C, Vacca A and Carratù MR Therapeutic targeting of the mTOR-signalling pathway in cancer: Benefits and limitations. Br J Pharmacol 171: 3801-3813, 2014. 